\title{
À la quête d'une territorialité en Aftût de Mbout sous la colonisation française (Mauritanie)
}

The Quest for Territoriality in Mbout Aftût under French Colonization

(Mauritania)

Cheikh Saad Bouh Kamara et Olivier Leservoisier

\section{OpenEdition}

Journals

Édition électronique

URL : http://journals.openedition.org/etudesafricaines/171

DOI : $10.4000 /$ etudesafricaines.171

ISSN : $1777-5353$

Éditeur

Éditions de l'EHESS

Édition imprimée

Date de publication : 1 janvier 2000

Pagination : 187-214

ISBN : 978-2-7132-1356-4

ISSN : 0008-0055

\section{Référence électronique}

Cheikh Saad Bouh Kamara et Olivier Leservoisier, « À la quête d'une territorialité en Aftût de Mbout sous la colonisation française (Mauritanie) », Cahiers d'études africaines [En ligne], 158| 2000, mis en ligne le 20 novembre 2013, consulté le 05 février 2021. URL : http://journals.openedition.org/ etudesafricaines/171; DOI : https://doi.org/10.4000/etudesafricaines.171 


\section{Cheikh Saad Bouh Kamara \& Olivier Leservoisier}

\section{À la quête d'une territorialité en Aftūt de Mbout sous la colonisation française (Mauritanie)}

Dès son arrivée dans l'Aftūt de Mbout, au tout début du Xx ${ }^{\mathrm{e}}$ siècle, le colonisateur français se trouve confronté à un problème d'organisation : comment contrôler les tribus maures nomades de la zone dont la vie se caractérise par une forte mobilité spatiale ? Toutes ou presque sont, en effet, en perpétuel mouvement qu'expliquent en partie la transhumance du bétail et l'insécurité due aux risques de razzias. Pour autant, chacune d'elles sait identifier la zone approximative qui correspond soit à son axe de parcours, soit à son aire d'influence, ce qui fit dire à E. Flandin (1908: 8) que « chaque tribu, chaque fraction connaît ses puits, ses terrains de culture, l'aire de parcours de ses troupeaux, les emplacements de ses campements. Elles reviennent à des époques déterminées dans les mêmes régions, si bien qu'on a à peu près la certitude de les rencontrer à dates fixes aux mêmes points ».

La mobilité était donc «disciplinée » à en croire cet administrateur. Cependant, l'utilisation d'un site était souvent éphémère puisque dépendante de la sécurité et des données climatiques. En outre, la nature politico-militaire du contrôle territorial en milieu maure ne permettait guère d'assurer une occupation durable des terroirs. Dans cet Aftūt de Mbout, dominé à l'époque par la puissante tribu guerrière des Idaw ‘iš, la jouissance du sol (pâturages, eau, redevances) était, en effet, fonction de l'aire d'influence ou des protections dont bénéficiait une tribu (voir carte Les émirats maures et les provinces de Fuuta Tooro page suivante). L'accès aux ressources pouvait, dès lors, être remis en cause selon l'évolution des rapports de force en présence.

Ces formes d'occupation et d'utilisation de l'espace, liées au nomadisme, étaient peu adaptées à la philosophie du colonisateur, pour qui l'assise de son pouvoir avait besoin de stabilité, de sécurité et d'organisation administrative. La solution au problème posé par la mobilité spatiale 


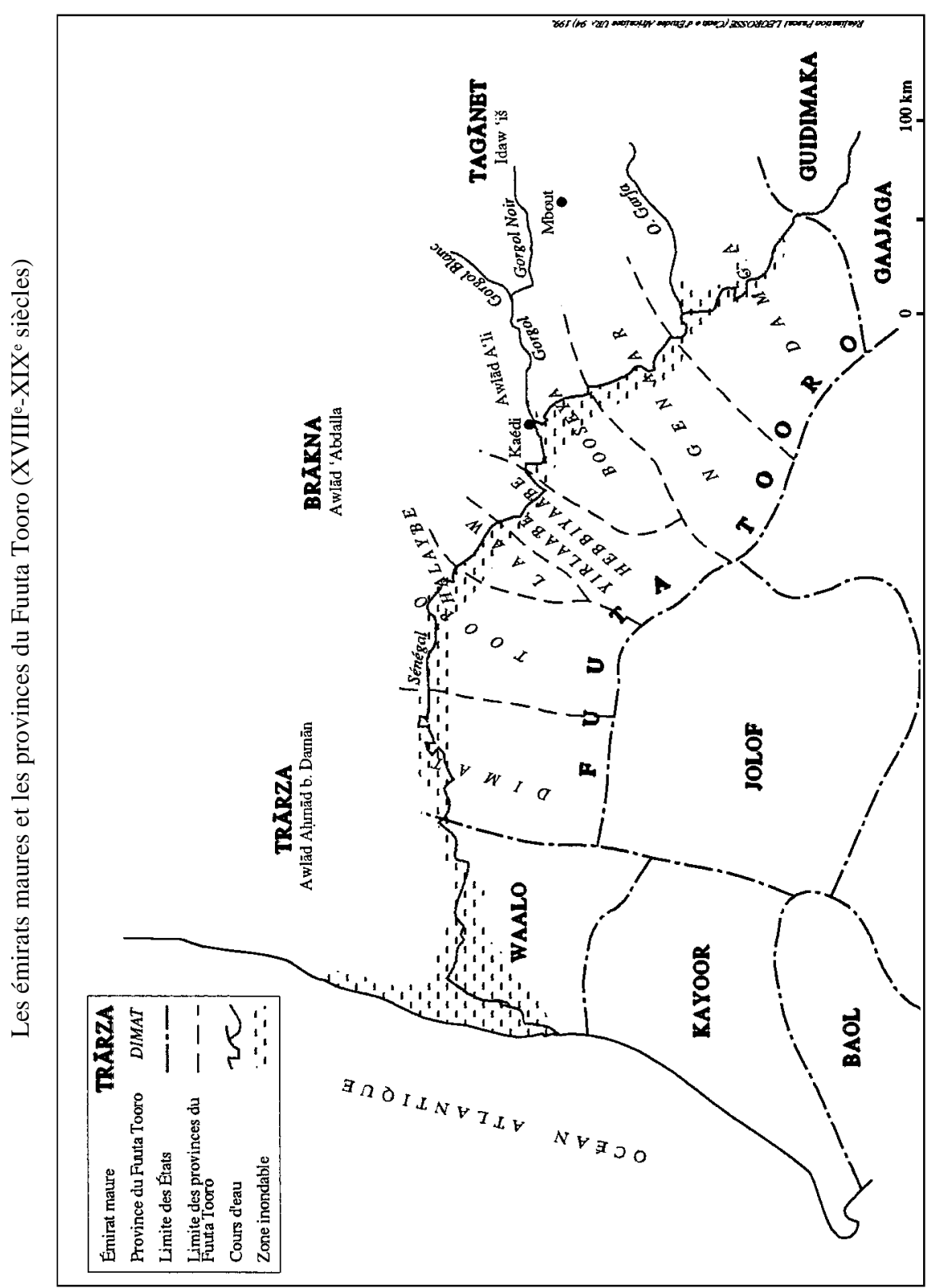


semblait donc passer par la diffusion d'un nouveau mode d'inscription territoriale. Celui-ci allait se traduire par le développement de l'agriculture et par la création d'un registre foncier censé inscrire les droits de chacun.

L'examen des modalités d'application des mesures prises par les autorités coloniales pour contrôler les populations retiendra notre attention afin de souligner les contradictions des politiques suivies et de comprendre comment les groupes concernés ont réagi à l'enregistrement des terres dans la région de Mbout. Mais il s'agira surtout d'analyser les conséquences de ces mesures sur la vie politique locale. On verra ainsi que, loin de fixer la carte tribale, le colonisateur a, au contraire, favorisé le développement de nouveaux rapports politiques en imposant progressivement de nouvelles formes d'inscription territoriale passant par la mise en valeur agricole. C'est dans ce contexte de transformations que certaines tribus maraboutiques, comme celle des Šurvā de Mbout, parviendront à s'implanter durablement dans la zone en se constituant un important domaine foncier. L'incitation à l'agriculture aura également des répercussions sur l'évolution des rapports sociaux. Certains groupes serviles chercheront ainsi à acquérir des biens fonciers, ce qui n'ira pas sans poser aux Français le problème de la détermination des droits de propriété et de l'attitude à adopter vis-à-vis de l'esclavage.

\section{Du contrôle des populations par l'autorité coloniale}

Deux mesures principales furent prises par l'administration coloniale pour contrôler les populations. La première consista en la création de limites administratives à partir des anciennes zones de parcours. Chaque tribu, chaque fraction, fut alors inscrite sur le registre d'un poste, d'une résidence ou d'un cercle. Cette division administrative présentait le double avantage de surveiller les tribus nomades jugées les plus turbulentes et de faciliter la perception de l'impôt. La deuxième mesure visa à favoriser l'agriculture. Elle devait permettre, selon les termes de l'administrateur Adams, «d'avoir à côté du nomade, souvent presque insaisissable, un élément sédentaire sur lequel on peut toujours compter $»^{1}$. Mais, outre ces préoccupations d'ordre politique, elle devait répondre à des intérêts économiques : la mise en valeur agricole devait accroître les revenus de la future colonie. Sur ce point, l'Aftūt de Mbout, formé d'une vaste plaine inondée par deux grands affluents du fleuve Gorgol : le Gorgol noir et le Gorgol blanc, alimentés à leur tour par de nombreux oueds (dont ceux de Mbout, Farkāne et Badyāb), apparut très vite comme une zone de prédilection. Coppolani n'a d'ailleurs pu se passer d'évoquer le potentiel agricole de cette région.

1. Rapport politique de la Mauritanie, $1^{\text {er }}$ semestre 1905, Archives nationales de Mauritanie (ANM). 
«M'Bout est une immense plaine traversée par le marigot du même nom généralement cultivée. C'est un pays d'une fertilité prodigieuse appelé à un très bel avenir au point de vue agricole et commercial en raison de sa situation exceptionnelle sur la principale voie d'accès au Tagant au bord d'un marigot où l'on trouve de l'eau et du poisson d'un bout de l'année à l'autre (fait connaître M. le résident du Gorgol). Tout permet d'ajouter foi à ces affirmations lorsque l'on a pu constater, comme nous avons été à même de le faire, que les terrains de culture de M'Bout et de Mouit alimentent en grains une grande partie des populations des vastes régions occidentales du Tagant $»^{2}$.

Soucieux à la fois de fixer les populations pour mieux les contrôler et accroître la production agricole, le colonisateur favorisa l'implantation de villages de culture (adabwa sing. adabay) habités par les harāțīn, les groupes serviles de la société maure ${ }^{3}$.

L'ensemble de ces décisions prises par la France ne manqua pas d'avoir des répercussions sur le contrôle foncier. Les nomades devaient désormais vivre avec la nouvelle notion de frontière imposée par le colonisateur. Pour se déplacer, il fallait que la fraction, par le biais de son chef, fasse connaître à l'autorité son intention, son itinéraire et la durée du séjour. Aussi, l'autorité émirale ou tribale qui s'étendait sur des surfaces parfois très vastes, s'est vue réduite. Le coup le plus dur fut l'écrasement ou presque de la turbulence des guerriers (hassān). Leur pouvoir foncier, soutenu par le sabre, tomba en désuétude à la suite de la «pacification » imposée par le colonisateur et des nouvelles formes d'inscriptions territoriales privilégiées. Celles-ci reposent fondamentalement sur la fixité et l'exploitation ${ }^{4}$.

Le principe de «liberté des pâturages », appliqué par les Français, affaiblit davantage le pouvoir des ḥassān, en remettant en cause les différents droits de passage dont bénéficiaient certaines chefferies. Cette règle de « libre-pâturage » n'ira d'ailleurs pas sans affaiblir l'argument de ceux qui revendiquaient des droits sur la terre au nom du nomadisme. Ainsi, progressivement, les bases sociopolitiques du contrôle foncier se sont dissoutes. Cette évolution résulte, pour une large part, de l'opposition entre deux conceptions de la territorialité. Alors que pour les tribus maures, les frontières étaient ouvertes et soumises à fluctuations selon le jeu des rapports

2. Archives d'Outre-Mer (АOM), SG Mie IV 1. Rapport du Secrétaire général des colonies en mission, délégué du Gouverneur général en pays maures, à M. le Gouverneur général de l'AOF, St-Louis.

3. La spécialisation des activités dans la société maure traditionnelle écarte en principe les bidān (nobles guerriers ou marabouts) du travail de la terre réservé aux populations serviles. La production agricole de cette main-d'œuvre servile venait en complément des activités pastorales de leurs maîtres bidān.

4. Signalons que les changements ont été particulièrement importants pour les harāṭ̂n des tribus guerrières. Comme le souligne 'Uțmān w. Muhtār, chef général des Šrātīt : «Avant l'arrivée des Français les haräạtīn nous suivaient. Les maîtres et les harāțīn se déplaçaient ensemble, nos harāâtịn n'étaient pas des cultivateurs ils nous suivaient dans les combats, c'étaient les tribus maraboutiques qui avaient des adabwa. Pour nous cela s'est développé pendant la colonisation. » Entretien, Mbout, le 3 mars 1992. 
politiques, les Français cherchèrent à imposer une autre conception de l'espace qui passait par la délimitation physique précise d'un territoire. Dans le premier cas, les zones soumises au droit de protection justifiaient des droits territoriaux. Dans le second, la légitimité foncière procédait de l'exploitation de terres cultivables et de leur immatriculation. Ces divergences dans la manière de penser et d'occuper le territoire renvoyaient, en réalité, à une conception différente de la souveraineté (Sautter 1982).

Si les tribus guerrières eurent particulièrement à souffrir des transformations engendrées par la colonisation, les tribus maraboutiques firent preuve, quant à elles, d'une meilleure adaptation. Traditionnellement attachées aux activités économiques (commerce, agriculture), certaines d'entre elles surent tirer profit du développement des rapports marchands et de la mise en place d'un registre foncier.

\section{Modalités d'application d'enregistrement des terres}

En Aftūt de Mbout, la politique d'immatriculation a été lancée suite à la promulgation du décret du 24 juillet 1906 organisant le régime de la propriété foncière dans les colonies et territoires relevant du gouvernement général de l'Afrique occidentale française (AOF). Ce décret reconnaissait les droits coutumiers tout en donnant la possibilité aux populations d'accéder à la propriété privée. Ainsi, l'individu qui souhaitait obtenir un titre foncier privé devait impérativement avoir recours au système de l'immatriculation qui convertissait son droit coutumier en un droit de propriété incommutable. Cette procédure individuelle n'eut aucun impact tant elle était contraire au principe de base du système de tenure traditionnelle du sol, selon lequel la terre est un bien collectif, inaliénable ${ }^{5}$. Conscient des limites d'application d'un tel décret, le colonisateur ne tarda pas à assouplir sa position en proposant un compromis qui permettait de conserver les intérêts des populations. Ainsi, les collectivités tribales n'ayant pas, selon le droit français, de personnalité juridique, il fut décidé que leurs terres pouvaient être enregistrées au nom du chef ou d'un représentant des dites collectivités ${ }^{6}$. Le décret du 8 octobre 1925 instituant un mode de constatation des droits fonciers des «indigènes » en AOF confirma davantage la reconnaissance des droits fonciers coutumiers. Ce nouveau texte juridique créait le régime de la transcription, qui permettait aux populations de faire constater et inscrire leurs terrains sur les livrets fonciers. À la différence du régime de l'immatriculation tel qu'il fonctionnait jusqu'à cette date, celui de la transcription n'entraînait aucune conséquence sur les biens fonciers des populations, puisqu'il ne s'agissait que d'une procédure d'enregistrement

5. Les oueds sont ainsi propriété collective de la tribu ou de la fraction. L'exploitation individuelle des terres n'est possible que dans le cadre de ces entités collectives auxquelles on appartient soit par filiation soit par adoption.

6. Archives nationales françaises (ANF) : 1908, L31 200MI : 1204. 
donnant à leurs détenteurs un certificat administratif de reconnaissance de leurs droits. Le décret du 26 juillet 1932, portant réorganisation du régime de la propriété foncière en AOF, confirmait et garantissait les propriétés inscrites sur les registres qui théoriquement ne pouvaient plus faire l'objet de contestations ${ }^{7}$.

En instaurant un nouveau mode de reconnaissance des droits fonciers, le colonisateur pensait fixer les populations sur leur terroir tout en mettant un terme aux situations conflictuelles. En réalité, les titres concédés furent loin d'être immuables et les compétitions entre les différents prétendants d'un site eurent tendance à s'accentuer. L'immatriculation comme source de légitimité se voyait opposée aux droits coutumiers. L'exemple du litige d'appropriation des terres de l'oued Badyāb entre tribus Šurvā et Tajakānit est sur ce point révélateur des limites de l'efficacité du registre foncier. Il montre par ailleurs la grande flexibilité dans la manière d'agir des intéressés. Un représentant des Survā raconte :

«Badyāb a été défriché par les Šurvā. Au début de la colonisation, tous les chefs devaient se rendre aux bureaux du poste administratif à chaque matin au risque de subir un emprisonnement de 15 jours. Al- ${ }^{\mathrm{C}}$ Abqary, chef des ar-Mazine (Tajakānit) était basé à al-Qabra. Tous les jours il se rendait à Mbout à dos de cheval. Trouvant que le voyage quotidien est pénible et rassuré par les excellents rapports d'amitié et de parenté qui le liaient à Hašim (chef des Survā), il lui demande de lui céder Badyāb pour qu'il y établisse son quartier, donc non loin de l'administration. Hašim céda Badyāb à al- ${ }^{\mathrm{c}} \mathrm{Abqary}$ et a déclaré la cession auprès de l'autorité pour se départir de l'impôt dû au dit terrain. Al-'Abqary prit alors l'impôt en charge pendant quelques années mais remarquant que la terre n'était que peu exploitée puisque n'attirant qu'une faible main-d'œuvre, il la céda à nouveau à Hašim. Après la mort des deux hommes, les Šurvā avaient à leur tête Jacfar W. Hašim et les ar-Mazine, le très jeune Zayn W. al- ${ }^{\mathrm{c}}$ Abqary. Ce dernier envoya une délégation auprès de Ahl Hašim (Šurvā) pour redemander Badyāb. Jacfar, ' Utmān et Šarīv, les trois membres les plus âgés de la famille se sont réunis pour étudier la demande. Elle fut rejetée par 'Utmān et Šarīv arguant que c'est leur oued, qu'ils l'ont déjà immatriculé à leurs noms et qu'il n'est pas question de le céder. Ja'far rétorqua en disant que si les ar-Mazine leur demandaient autre chose (plus précieux), ils l'auraient ${ }^{8}$.

Du côté des Tajakānit, cette version est tout simplement inversée. Ils s'appuient sur le registre foncier de Mbout, indiquant que Badyāb est enregistré au nom des ar-Mazine et porte l'indication historique «cultivé avant

7. M. Villasante Cervello (1989: 213-248) a indiqué pour la région de l'Assaba que la propriété foncière fut fixée, essentiellement, entre 1932 et 1936, période durant laquelle la majorité des titres fut attribuée. Précisons que jusqu'en 1913, Mbout faisait partie du cercle du Gorgol. Par la suite, l'administration française jugeant que les populations de Mbout avaient plus de liens avec celles de Kiffa décida, par arrêté du 12 novembre 1913, de transférer le secteur de Mbout dans le cercle de Kiffa (ANF 9G33 : 200 MI 850). Mbout est repassé dans la région du Gorgol en 1969 sous la présidence de Moktar ould Daddah, au moment de la création des régions.

8. Entretien H. W. 'Uṭmān, le 30 novembre 1990. 
l'occupation ». Mais en dépit de cet enregistrement, l'état d'occupation a changé. Le terrain est divisé en deux, entre les Šurvā et les Tajakānit.

Nous pouvons nous demander comment les ar-Mazine ont perdu ce qui semble avoir été leur domaine ? Comment les Survā gardent-ils une partie seulement d'un terrain qu'ils prétendent avoir défriché et prêté ? Ces questions n'auront jamais de réponses puisque la confrontation entre une légitimité coutumière et celle tirée du droit administratif est tout à fait stérile, les arguments des Tajakānit pouvant être infirmés par les Šurvā et vice versa. Et ce qui ressemble à un droit acquis par voie de défrichement dans l'esprit des Šurvā n'a pas réussi à surpasser ce qui est le droit d'immatriculation des Tajakānit.

Les Šurvā sont pourtant les premiers à déclarer leurs terres en 1909 (Oued Amour). Les Tajakānit, quant à eux, n'ont obtenu leur première autorisation que trois ans plus tard (à Tisilite al-Bayza et Tisilite al Hazra). Les Šurvā ont-ils omis de déclarer Badyāb parce que prêté ou parce qu'il ne leur appartenait tout simplement pas ? Les Tajakānit ont-ils profité du prêt pour officialiser leur mainmise en déclarant le terrain auprès de l'autorité coloniale, ce qui rendrait mensongère la mention «cultivé avant l'occupation »? Aucune réponse ne peut encore une fois être avancée. Cependant, signalons que le terrain en question étant sis dans l'aire de mouvance des deux tribus, chacune d'elle pouvait le revendiquer en totalité ou en partie. L'oued est assez grand et il n'est pas exclu que des éléments des deux tribus y aient pâturé et/ou cultivé à un moment de leur histoire, ce qui, à leurs yeux, est une forme de territorialité sur la dite zone. Les deux tribus étant fortement liées par des liens familiaux, il ne leur restait que la voie du compromis pour mettre un terme à ce litige.

Il reste qu'au moment de l'immatriculation, chaque tribu voulait avoir le maximum de propriétés. Les marquages par le biais du nomadisme, agriculture, creusement de puits, clôture, etc., étaient signalés par les différents prétendants. À l'établissement des autorisations, l'administration coloniale pratiquait des enquêtes conformément aux lois en vigueur et autant que faire se peut, elle essayait de confirmer ou d'infirmer les revendications foncières des demandeurs. En cas de litige extra-tribal, elle tentait, à la lumière de quelques témoignages, d'appliquer la loi française relative à l'occupation dite « évidente et permanente». Mais cette administration était souvent responsable des situations conflictuelles. Les querelles étaient fréquemment occasionnées par le manque de précisions dans les délimitations des terrains concédés. Les concessions qu'ils attribuaient étaient la plupart du temps précipitées et ne tenaient guère compte des réalités du pays :

«Beaucoup de terrains ont été accordés par l'administration depuis plusieurs années. [...] La situation de la plupart des terrains accordés est mal définie. Le même terrain a quelquefois été concédé sous deux noms différents à deux tribus. Il s'ensuit des complications nombreuses ${ }^{9}$.

9. Rapport politique du cercle du Gorgol du quatrième trimestre 1913. ANM : dossier E1/48. 
L'imprécision qui dominait dans ces donations était souvent due à l'impartialité des administrateurs. Il n'était pas rare en effet que ces derniers acceptent avec complaisance les larges revendications foncières de certaines communautés, avec lesquelles ils souhaitaient pactiser. Cette attitude conciliante bénéficia tout particulièrement aux tribus maraboutiques Tajakānit et Šurvā. Ainsi, dès 1913, le Résident de Mbout écrivait au commandant du Gorgol au sujet du chef des Šurvā : «Hachim est le premier chef à se soumettre aux Français dans la région de M'Bout [...]. Les premiers soumis sont traités avec plus de déférence et de cordialité ${ }^{10}$. Et le même texte ajoutait au sujet de ad-Dāh W. Zyād des Tajakānit : «Continue à servir avec la plus grande loyauté. Renseigne le Résident sur tout ce qu'il apprend. Lui accorder les faveurs qu'il demande ${ }^{11}$. Il est clair que la partialité s'est mise de la partie.

À la décharge des administrateurs, il faut reconnaître la difficulté de leurs tâches. Les terres étaient souvent revendiquées par plusieurs groupes, dont la légitimité des droits était loin d'être évidente. En outre, les conflits n'étaient jamais définitivement résolus car la stratégie la plus fréquente utilisée par les populations était de revendiquer les droits sur la terre à l'arrivée d'un nouvel administrateur. Les plaintes étaient inlassablement réitérées dans l'espoir de voir un jugement plus favorable. Les hésitations de l'administration coloniale en matière de donations de terrains confortaient les populations dans leur démarche, qui savaient qu'elles pouvaient obtenir un jour gain de cause.

Nous savons, par ailleurs, que l'administration souffrait de plusieurs handicaps. Les moyens matériels et techniques faisaient défaut. La méconnaissance géographique du terrain rendait également plus difficiles les opérations de délimitation. À titre d'exemple, l'oued que déclare une tribu peut s'étendre sur plusieurs kilomètres, changeant de nom et parfois de propriétaire en plusieurs de ses points. C'est le cas de l'oued de Mbout qui, avant de se jeter dans le Gorgol noir dont il est un affluent, bute sur Ndūmrāte et ar-Rakna. C'est aussi le cas de l'oued Kow, autre affluent du Gorgol noir et qui est divisé en flik (parties, sing. filka) ayant chacune ses propriétaires.

En déclarant un oued, pouvait-on aspirer à se l'approprier en totalité même s'il n'est exploité et contrôlé qu'à moitié ou moins ? Oui, répondaient sans ambages les postulants favoris, non, rétorquaient leurs rivaux. Mais la règle générale voulait que le terrain soit mis en valeur même si, lors de la première déclaration, le colonisateur fit preuve d'une souplesse parfois remarquable dans le but de promouvoir l'activité agricole.

Les petits ravins appelés «Silaḥ» (sing. Salha) dont la crue alimente les oueds dans lesquels ils se jettent, furent également sources de confusion et de conflits. Ces ravins, très souvent négligés comparativement à l'oued

10. ANM, E1 42. Correspondance du 3 décembre 1913.

11. AOM, 14 MI 853. M'Bout, 3 janvier 1914. 
principal, revenaient-ils de droit au propriétaire dudit oued ou constituaientils un autre territoire distinct? Selon M. W. al Haj, «le défrichement comprend les Silah, mais ces terrains sont individuels contrairement à l'oued qui est propriété de toute la tribu et où le propriétaire de la Salḥa peut avoir une parcelle » ${ }^{12}$. Mais si une personne «propriétaire »d'une Salha n'a pas une grande famille lui permettant d'exploiter les deux terrains, il est à penser que le terrain sis dans l'oued soit prioritaire. Il reste que ces problèmes d'attribution et de délimitation des droits lors des concessions ne manquèrent pas de susciter des tensions.

«En 1932, le Résident de M'Bout autorisa, sans enquête préalable, le maure Sidi Ould Eleya à cultiver la région arrosée âr l'oudeilout, bien que depuis vingt ans un affluent de ce marigot "le Fedra" fut défriché et exploité par les ahel Salah. Cette autorisation fut l'objet de nombreuses déclarations et provoqua du désordre ${ }^{13}$.

Complaisance, complicité, erreur de jugement ou manque de données suffisantes ? En tout cas, plusieurs opérations d'affectations et de désaffectations se produisirent. L'affaire Demba w. Guelay (Litāma), survenue au début $\mathrm{du} \mathrm{Xx}^{\mathrm{e}}$ siècle, en est un parfait exemple. Ce chef de Djajibine avait en effet revendiqué, au nom d'une donation faite par un administrateur, la propriété des terres situées à proximité de son village, lesquelles étaient cultivées par des Šurvā et des Soninké. Le litige semblait difficile à résoudre car un rapport de l'époque précisait à son sujet: «Le libellé des papiers qui lui ont été remis, l'a amené à croire que sa concession était personnelle $»^{14}$. Les choses se compliquaient d'autant plus que les Soninké du Guidimaka avaient été autorisés, en 1906, par leur résident à cultiver sur l'oued de Djajibine. Finalement, devant les tensions que souleva cette affaire, l'administration adopta en 1910 l'une des deux mesures principales qu'elle finissait habituellement par admettre lorsqu'elle ne prenait pas ouvertement parti : le partage des terrains entre les trois parties concernées fut préféré à la neutralisation des terres. Malgré ce jugement, Demba w. Guelay, en 1926, en réclamait toujours la propriété, arguant qu'il n'est pas seulement le premier à les avoir occupés avant l'arrivée des Français, mais que «pendant sa jeunesse, il a beaucoup travaillé pour la France » ${ }^{15}$.

On le voit, la création d'un registre foncier qui devait en principe refléter l'état concret de l'occupation de l'espace a en réalité redéfini un certain nombre de propriétés. Elle a également, contrairement à ce qui était annoncé officiellement, exacerbé les rivalités, chaque partie en litige souhaitant obtenir gain de cause et conserver ses droits. Cependant si l'immatriculation est considérée comme nouvelle base de légitimité, elle l'est surtout pour

12. M. W. al Haj, entretien du 13 décembre 1990.

13. Rapport politique de 1940 du commandant de cercle Castagnet, ANM : dossier E2 100.

14. Mbout lettre du 3 avril 1910, anonyme, dossier E1 7.

15. ANM, O 1. Correspondance Résident de M'Bout, 20 avril 1926. 
celui qui en bénéficie et non pour la partie lésée. En ce sens, la procédure d'immatriculation n'apparaît pas comme une fin en soi mais est une source de légitimité parmi d'autres (droit de défrichage, de protection...) que l'on utilise ou non selon les intérêts du moment. La meilleure illustration de cette marge de liberté des populations à l'égard du droit du colonisateur reste sans conteste l'exemple des arrangements entre tribus visant à redéfinir une répartition territoriale, en dépit de ce qui avait pu être préalablement inscrit au cadastre. Le cas de Badyāb sur lequel nous nous sommes attardés est sur ce point éloquent.

$\mathrm{Au}$ vu de ces premiers résultats, il semble donc que l'utilisation du registre foncier dans la région de Mbout ait pris la voie d'un compromis entre l'administration française soucieuse de contrôler les populations, et certaines tribus maures qui y trouvaient un argument supplémentaire à faire valoir en cas de litige tout en s'assurant un contrôle territorial. Les Šurvā de Mbout surent en tirer le meilleur profit.

\section{Nouvelle donne politique : I'émergence des Šurvā}

Si les Šurvā ont profité des nouvelles formes d'inscription territoriale préconisées par le colonisateur, c'est d'abord parce qu'ils furent l'une des rares tribus attachées à l'agriculture. Certes, l'élevage était étroitement associé à cette activité, mais il ne remit jamais en cause leur vocation agricole, qui leur valut le surnom de Šurvā de Butali (Šurvā du maïs). C'est d'ailleurs en tant qu'agriculteurs et non comme conquérants qu'ils effectuèrent, à la fin $\mathrm{du} \mathrm{XIX}^{\mathrm{e}}$ siècle, leur déplacement des rives du fleuve Sénégal à l'Afūt de Mbout. Le rappel de leur migration mérite d'être signalé afin de mieux comprendre comment une petite tribu maraboutique a pu acquérir, dans un laps de temps relativement court, un certain nombre de territoires et asseoir ainsi une domination politique toujours actuelle dans le département de Mbout $^{16}$.

Segmentarité et territorialité chez les Šurvā de Mbout

Les Šurvā ont donc pénétré en Aftūt de Mbout après avoir séjourné sur les rives du fleuve Sénégal. Leur présence dans cette partie du pays remonte à l'époque de leur ancêtre Sīdi Muḥammad w. Mulāy Hašim, plus connu sous le nom de Sīdi Šarīv, qui fut le premier à s'établir sur le territoire mauritanien (voir la Généalogie simplifiée des Šurvā de Mbout ci-après). Venu à la fin du XVIII ${ }^{\mathrm{e}}$ siècle de Touat dans le sud du territoire algérien, il s'installa dans l'Azouad (Hodh) auprès de la tribu des Kunta, puis se

16. Depuis l'Indépendance, les mandats de maire et de député sont en effet entre les mains des Survā. L'actuel député maire de Mbout n'est autre que Ma-l-'Aynin de la fraction des Hašim. 


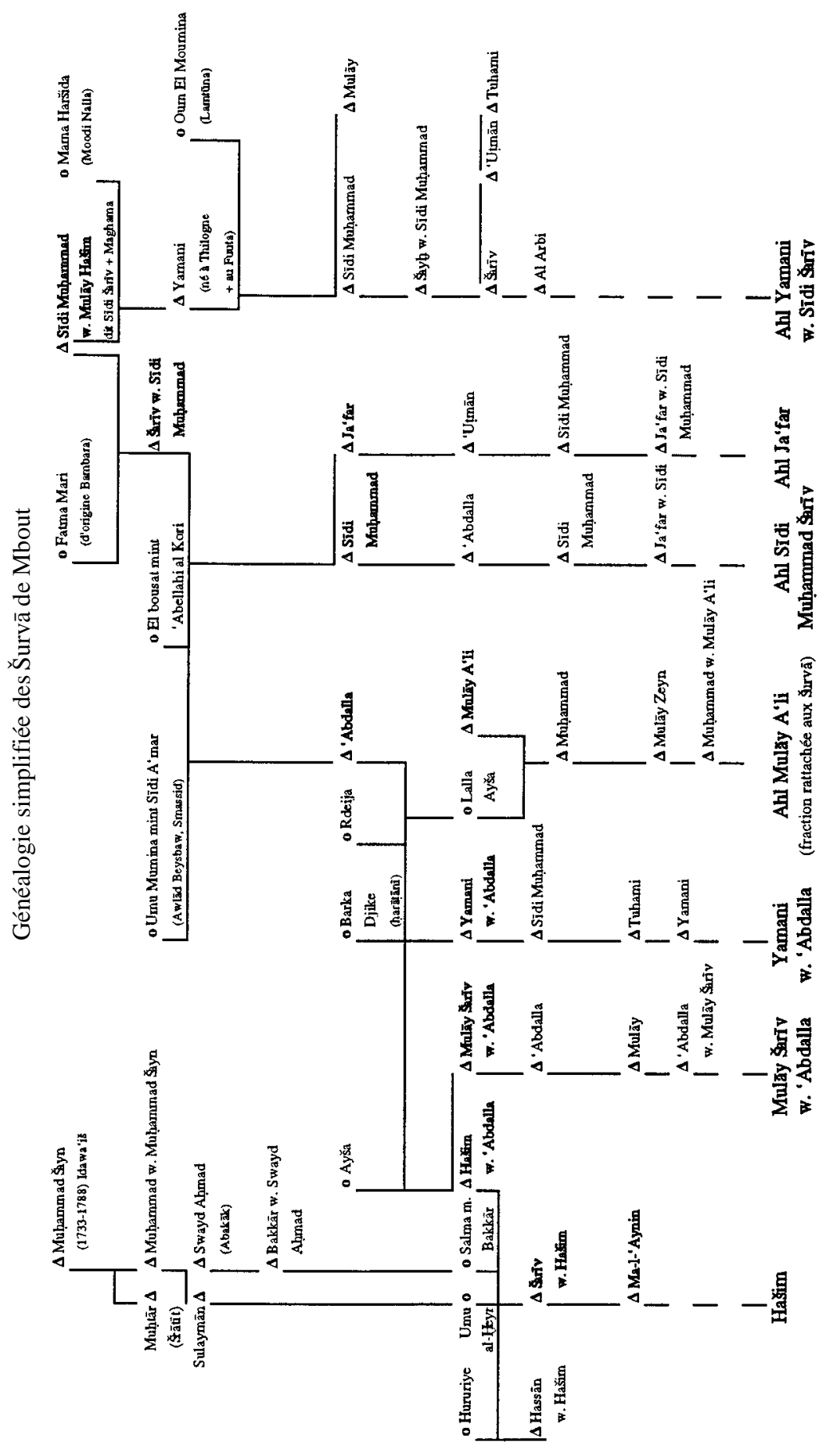


rendit après plusieurs étapes au Fuuta Tooro ${ }^{17}$ où il rencontra l'almaami Abdul Kaader à Kobillo et à Thilogne. Celui-ci lui aurait concédé, en signe de gratitude, des terres de culture appelées Modda sur la rive droite du fleuve Sénégal près de Maghama, qui demeurent jusqu'à présent sous le contrôle des Šurvā.

Fort de ces liens avec le Fuuta, Sīdi Šarī ${ }^{18}$ s'installa à Maghama (connu à l'époque sous le nom de Kumballi) pour y passer le restant de ses jours. À sa mort, ses deux fils Yamāni et Mulāy Šarīv lui succédèrent tour à tour. Le premier choisit, comme son père, de se maintenir dans le Fuuta au bord du fleuve Sénégal ${ }^{19}$. Le second manifesta, quant à lui, son désir de remonter vers l'intérieur du pays, en direction de l'oued de Kow qui était alors inhabité $^{20}$. Les harātīn des Šurvā y défrichèrent les terres.

Kow fut donc le premier site fréquenté par les Šurvā après Maghama. Mais les terres de Modda ne furent pas abandonnées pour autant. Pendant la saison sèche, ils y descendaient pour récupérer une partie du mil cultivé par leurs harāținn. Durant l'hivernage, vers le mois d'août, ils remontaient à Kow pour la mise en culture de leur oued.

Malgré sa volonté de pénétrer à l'intérieur des terres, Mulāy Šarīv, comme son fils 'Abdalla, qui lui succéda, restèrent attachés au Fuuta Tooro où ils furent tous deux enterrés. À la mort d'Abdallah, les Šurvā vont véritablement amorcer leur progression vers le nord, lorsqu'à la suite de querelles de succession entre son fils et ses frères, la tribu se scinda en trois fractions : les Hašim w. 'Abdalla, les Sīdi al-Ja'far et les Sīdi Muḥammad w. Yamāni. La création de ces fractions marqua une prise de distance à l'égard du Fuuta Tooro.

C'est ainsi que la fraction des Hašim (le fils d' 'Abdalla qui s'imposera chef général de la tribu sous la colonisation) s'est répartie principalement sur les oueds de Kow el Vogani, de Djajibine et de Mbout (voir carte Le contrôle foncier des Šurvā de Mbout). Son arrivée à Mbout serait intervenue vers 1890, peu de temps avant celle des Français au début du siècle ${ }^{21}$.

Les Sīdi al-Ja 'far qui regroupaient les deux frères d' 'Abdalla (Sīdi Muḥammad et Ja'far) ont, quant à eux, cultivé ensemble un endroit appelé Nabam dans les Agueilatt et se sont installés à Bathat. Mais à la mort de

17. Situé dans la moyenne vallée du fleuve Sénégal, le Fuuta Tooro constitue l'ancienne entité politique des Haalpulaar'et qui, à la fin du XVIII ${ }^{\mathrm{e}}$ siècle, fut dirigée par le régime des Almaami.

18. Il épousa deux femmes noires, Fatma Mari, esclave d'origine bambara, dont certains Šurvā pensent qu'elle fut donnée par l'almaami, et Mama Haršida, du clan des Kan des Moodi Nallaykoo6e.

19. Il fut enterré à Hatrengu au pied d'une colline située entre les villages de Dao et de Maghama.

20. Mulāy Šarīv occupa Kow el Vogani.

21. Selon Hassān w. Monane (entretien le 9 octobre 1991), ce n'est qu'en 1889 que les Šrvā ont quitté Kow pour se rendre à Mbout. Selon Deigat, harāțīn de l'adabay Šurvā de Mbout, ils ont précédé les Français à Mbout d'environ vingt ans. À cette époque, ils vivaient dans des campements (vrigh). 
Le contrôle foncier des Šurvā de Mbout

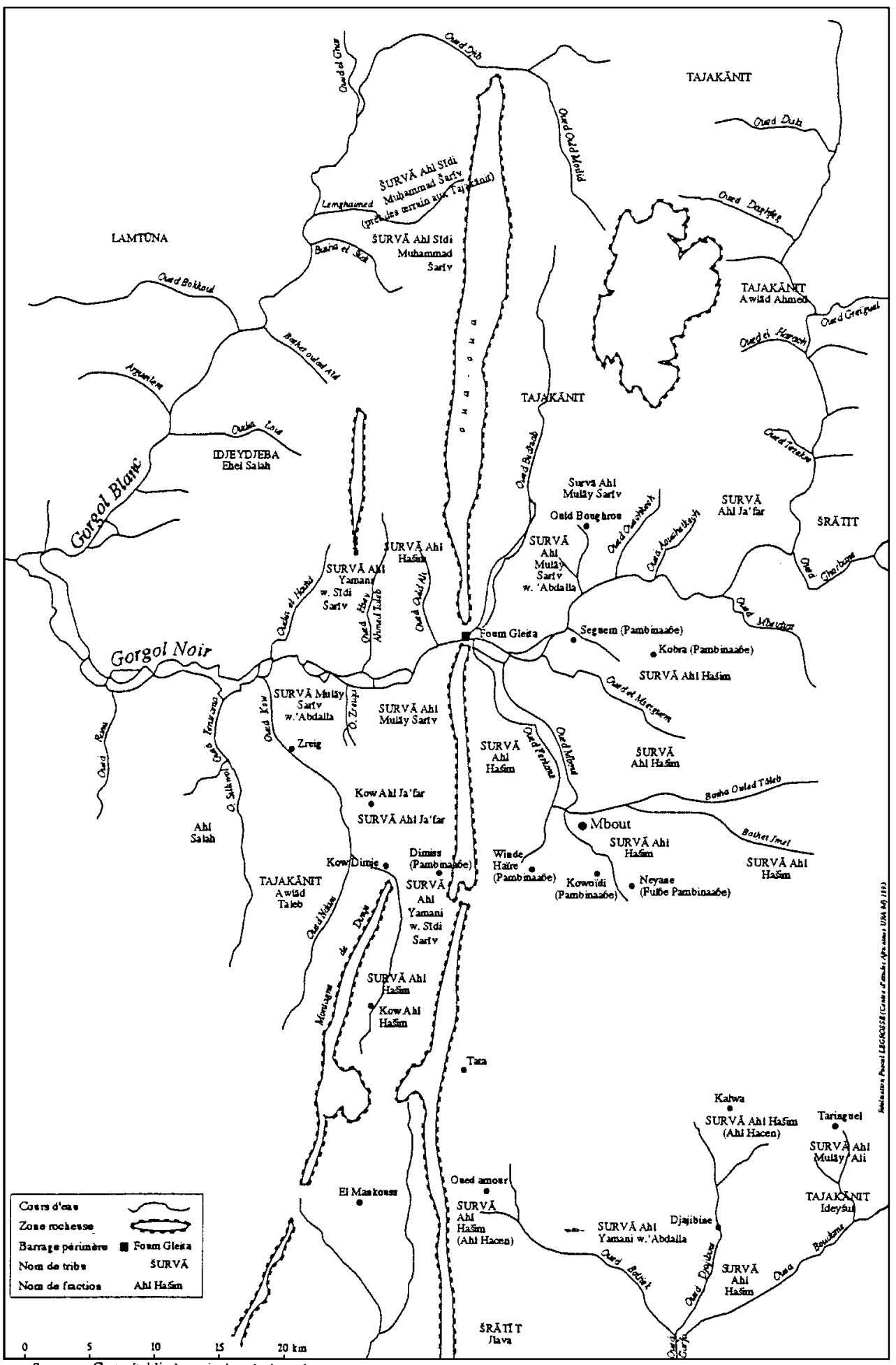


Sīdi Muḥammad, son fils 'Abdalla a créé sa propre fraction et est parti à Kow Vogani puis à Ndam. De son côté, Ja'far est venu s'installer à Kow el Ja'far, ses fils iront à Ouechkech.

Enfin les Sīdi Muhammad w. Yamāni sont partis là où se déverse le Gorgol blanc, à Chelkra w. Yamāni. Puis ils sont allés à Hsey Aḥmad Taleb avant de revenir à Kow el Yamāni.

Par la suite, deux nouvelles fractions issues des rangs des Hašim w. 'Abdalla seront créées en raison d'un différend entre Hašim et son frère Mulāy Šarīy. Ce dernier est parti s'installer à Ouechkech. Au même moment, leur frère Yamāni w. 'Abdalla a fait scission et s'est installé à Bothiek.

Au total, la tribu des Šurvā compte donc six fractions (fahd) dont les membres, descendants de l'ancêtre commun Sīdi Šarīv, se sont répartis sur différents oueds de la région ${ }^{22}$. Encore fallait-il qu'ils puissent les mettre en valeur lorsque l'on sait qu'en cette fin de $\mathrm{XIX}^{\mathrm{e}}$ siècle — époque où s'amorce la remontée vers le nord des Šurvā — le puissant émirat guerrier des Idaw 'iš exerçait son contrôle sur le Tagant, l'Assaba et la rive du fleuve Sénégal de Kaédi à Bakel. Mais les Šurvā disposaient de plusieurs atouts pour se maintenir sur place.

\section{Les facteurs favorables à la progression au Nord des Šurvā}

Plusieurs facteurs ont rendu possible l'installation des Šurvā au sud du Gorgol noir. En premier lieu, le contrôle territorial exercé par les Idaw 'iš était moins important dans cette zone que dans le nord de l'Aftūt ${ }^{23}$. Les Šurvā ont ainsi pu s'installer aux marges des pôles dominants que représentaient l'émirat du Tagant et le Fuuta Tooro. En second lieu, les Šurvā ne constituaient qu'une petite tribu et avaient la réputation de marabouts pacifiques. À la différence d'autres tribus maraboutiques qui n'hésitaient pas à prendre les armes, ils surent rester fidèles à leur réputation. Le troisième facteur favorable à leur implantation est lié à leur politique matrimoniale particulièrement active auprès des tribus guerrières qui leur permettait de bénéficier de protections. Parmi les mariages les plus marquants, figurent ceux de

22. À côté de ces six fractions dont les membres sont unis par filiation patrilinéaire, d'autres fractions ont été intégrées aux Šurvā par adoption (walā), à la suite d'alliances politiques ou matrimoniales. C'est le cas des Ahl Mulay A'li, des Ahl'Aleya, des Ahl Hommeni d'origine Lamtūna ou des Ahl Saleh de la tribu des Idjeydjba (Brakna).

23. Dans la région du Gorgol, le contrôle des Idaw 'iš s'exerçait principalement dans le nord de l'Aftūt de Mbout, plateau au réseau hydrographique dense favorable aux cultures des oueds et aux pâturages. À l'est, la zone des Agueilatt (Monguel) était le domaine des tribus Lamtūna, Awlād A'li, et des Awlād Tahla qui exploitaient les affluents du Gorgol blanc. C'est au nord du Gorgol noir que se sont concentrés les Tajakānit (fraction Rmadin et Awlād Ahmad), suivis au sud par les Šrātīt et les Survā. 
Hašim avec Salma mint Bakkār la fille de Bakkār w. Swayd Aḥmad (l'émir du Tagant mort en 1905 dans sa lutte contre les Français), et avec Umu el Heyr, fille de Sulaymān w. Muḥammad Šayn, descendant de Muḥammad w. Muḥammad Šayn, l'ancien chef des Idaw 'iš. Enfin, leur inscription territoriale dans le sud du Gorgol noir fut assurée par les liens privilégiés qu'ils entretinrent avec le colonisateur. En 1908, l'administrateur Adam écrivait au sujet de Hašim : «Bien avant notre pénétration mais parce qu'il la prévoyait et qu'il se trouvait déjà en relation avec Matam, il se rallia à notre cause. C'est certainement par intérêt qu'il a agi mais l'intérêt n'est-il pas le lien le plus durable ? $»^{24}$. En 1912, on pouvait également lire sur sa fiche de renseignements : "A traité avec nous longtemps avant notre occupation de la Mauritanie. A toujours été un allié fidèle et a rendu de gros services $»^{25}$. Les Šurvā se sont ainsi transformés, avec la colonisation, en tribu autonome jouissant d'une grande considération dans les milieux administratifs. Il fallait donc pour cette entité grandissante et qui était déjà présente sur le terroir, faire valoir «ses droits». Le contact, relativement ancien, entretenu avec l'autorité coloniale n'a pu que faciliter leur tâche dans l'immatriculation des terres tribales dès que la procédure fut engagée par les Français.

Les Šurvā bénéficièrent également de la «pacification » imposée par la France. La sécurité étant assurée, leur implantation s'en trouva facilitée. Elle eut des conséquences importantes sur la segmentation et la mobilité de la tribu car chaque fraction pouvait s'éloigner des siens sans risque de dépossession. Ce fractionnement occasionna une occupation dite «segmentaire » qui se traduisit par l'existence d'une même tribu en plusieurs endroits différents. Certes, le phénomène était déjà visible avant l'arrivée du colonisateur à travers les nombreux campements, mais il est devenu plus remarquable avec l'introduction de la nouvelle façon de marquer le sol que fut la sédentarisation.

\section{L'assise du pouvoir territorial des Šurvā par la captation d'ḥarāțīn}

Les Šurvā ayant su saisir les opportunités qui s'offraient à eux pour s'implanter sur différents sites, il leur restait à trouver les moyens de les mettre en valeur. La tâche n'était pas simple car, lors de leur arrivée dans l'Aftūt, ils ne constituaient qu'une petite tribu. La réponse fut finalement trouvée par une politique de captation d'harātīn et de 'abìd. Leur acquisition se réalisa soit par dons, phénomène fréquent chez les familles maraboutiques

24. ANM, dossier E1/7. Il fut considéré comme un allié fidèle ayant toujours rendu de grands services au point qu'il fut l'objet d'une proposition pour l'obtention d'une médaille d'honneur pour services rendus à la France en décembre 1911. Lettre $\mathrm{n}^{\circ} 225 \mathrm{du}$ colonel commissaire du gouvernement général en date du 8 décembre 1911, ANF 9G42 : 200 MI 853.

25. АOM, 14 MI 853, $1^{\text {er }}$ juillet 1912 . 
auxquelles on offrait des captifs en échange de leur protection, soit par achat, soit à la suite de l'affiliation de certaines fractions, qui en s'intégrant aux Šurvā était accompagnée de leurs dépendants. Mais le plus grand nombre vint se réfugier sous leur protection après avoir fui leurs anciens maîtres, le plus souvent d'origine guerrière ${ }^{26}$. Ce phénomène se produisit essentiellement au début de la colonisation lorsque la pacification de la zone déstabilisa le pouvoir des hassān. La politique matrimoniale des Šurvā auprès des tribus guerrières (Awlād Mbārik, Idaw ‘iš, Litāma) n'a fait également que favoriser la captation d'harāțīn issus de leurs rangs, dans la mesure où elle limitait les risques de voir les anciens maîtres insister pour les récupérer.

L'utilisation de la main-d'œuvre servile fut donc cruciale dans la consolidation du contrôle territorial des Šurvā. Elle permit de mettre en valeur de nombreuses terres qui autrement seraient restées en friche. Mais, en outre, elle leur donna parfois la possibilité d'acquérir de nouveaux terrains. C'est, semble-t-il, ce qui se produisit à Wul 'Ali et à Gleita. Ainsi, en 1921, une partie de l'oued Wul 'Ali et de Laglayta fut attribuée aux Hašim alors qu'il s'agissait de zones contrôlées par les Tajakānit de la fraction ar-Mazin. Le registre foncier de Mbout est sans équivoque. Il stipule à propos de W. 'Ali : «Reçu des R’Madines en $1921 »$, donc cédés par ceux-ci. Et pour «Glaytat», on peut lire : «Avait appartenu aux R'Madines qui cessent de prétendre à ce terrain accordé en 1921. » Il reste à savoir pourquoi et dans quelles circonstances les ar-Mazine ont «renoncé » à ce qui semble être leur $\ll$ possession $»^{27}$.

Nous pouvons avancer l'hypothèse que les ar-Mazine n'étaient pas assez nombreux. On a vu, en effet, dans le cas de l'oued Badyāb comment le chef des ar-Mazine avait cédé les terres aux Šurvā en raison d'un manque de main-d'œuvre. Il n'est donc pas exclu que le même problème se soit posé à Laglayta, encore plus excentrique par rapport au fief de concentration de la fraction Tajakānit (à al-Qabra). Les Šurvā, géographiquement proches de la zone concernée et disposant de nombreux harạtịn, ont ainsi pu selon toute vraisemblance récupérer les terres et faire inscrire leurs droits sur le registre foncier. Leur loyalisme ancien et zélé envers l'autorité coloniale a dû également favoriser cette inscription ${ }^{28}$.

26. Les harātịn fuyant leurs maîtres recherchaient une protection auprès des Šurvā mais également parce que ces derniers étaient proches du poste militaire de Mbout.

27. Pourtant la zone de Laglayta, dans laquelle se trouve aujourd'hui le site du barrage de Fum-Laglayta, continue à être revendiquée aussi bien par les Šurvā, les Šrātìt et les Ahl AcLayya.

28. Les Šrvā se querellèrent par la suite. En 1953, Šarīv w. Hašim (de Ahl Hašim), Sidi Muhammad w. Ja ${ }^{c}$ far (de Ahl Ja ${ }^{c}$ far) et al-Yamāni w. Šarīv (de Ahl Mulay Šarīv) se sont trouvés en justice au sujet de la délimitation de leurs domaines respectifs. Après jugement, la zone de litige fut divisée en cinq parties. Les Ahl Hašim, ancienne fraction dirigeante, ont eu droit à deux parties, Ahl Mulay Šarīv, Ahl Jacfar et Ahl AcLayya ont bénéficié chacune d'une partie. 


\section{Élargissement du pouvoir clientélaire aux Peuls}

Si les Šurvā ont su se constituer une importante clientèle harāṭ̂̄n, leur pouvoir foncier leur a également permis d'exercer une emprise sur certains groupes peuls de plus en plus nombreux à s'infiltrer en Aftūt de Mbout, durant la première moitié $\mathrm{du} \mathrm{Xx}^{\mathrm{e}}$ siècle ${ }^{29}$. Dès 1914 , leur pénétration dans cette région est attestée par l'administrateur Antonin: «Le passage des Peulhs sur la rive droite ne fait que s'accentuer, en même temps que s'étend leur zone de parcours, elle atteint à l'heure actuelle la région de M'Bout, où j'ai rencontré plusieurs de leurs campements $»^{30}$. En 1950 , le rapport de Mbout indique que «les Fulbé Dieri cultivent le mil à Moinit, Lahrache, Goumal, Barkéol » ${ }^{31}$. Il en était de même pour Hsay Ahmad Talib, Dysky, Bidyane, Awaskas, Vinde Aéré, Laglayta. Bref, l'Aftūt dans sa grande dimension se trouve clairsemé d'éléments peuls. Au début des années 1950, l'administrateur Cabrol (1954) y estimait leur nombre à 8 000. En 1968, selon les cahiers des recensements administratifs, les Peuls représentaient près de 23000 personnes, soit environ $25 \%$ de la population du Gorgol (Santoir $1990: 9)^{32}$. Au début des années 1960, certains Fulbe jeeri auront même franchi le massif de l'Assaba.

Dans le cas des Fulbe jeeri, leur grand fractionnement politique les a empêchés d'affirmer une certaine indépendance face aux tribus maures de la région. Cabrol, dans ses rapports administratifs des années 1950, indiquait que les fractions peules étaient très dispersées et la plupart mal commandées. Cette faiblesse dans leur organisation politique explique que la plupart des Peuls ont dû recevoir des autorisations pour creuser des puisards (olgat) et pour utiliser des terres de pâturages et de culture (Hervouët 1975). Ce fut

29. Plusieurs raisons, déjà évoquées par F. BonNET-DuPEYron (1947), expliquent cette remontée progressive des Peuls. La première est celle de la richesse des pâturages qui contrastait avec ceux du Sénégal, arrivés à saturation, du fait de l'accroissement de population. Les deux conflits mondiaux qui intervinrent pendant deux périodes de forte sécheresse, incitèrent les Peuls à migrer à l'intérieur du pays à la recherche de meilleurs pâturages et avec l'espoir, entretenu par le moins bon encadrement administratif en Mauritanie, d'échapper aux réquisitions de bétail et aux impôts.

30. ANM, rapport politique annuel de l'administrateur Antonin, commandant de cercle du Gorgol, 1914 (dossier E1 48).

31. Les Peuls se distinguent selon leur mode de vie en deux grandes catégories communément appelées fulbe waalo et fulbe jeeri. Les premiers sont "semisédentaires ». Ils s'adonnent aux cultures de décrue (waalo, d'où leur appellation) et ne pratiquent qu'un élevage de petite transhumance. Les seconds "seminomades » vivent davantage au rythme de leurs troupeaux. Leur activité pastorale est complétée par la culture sous pluies appelée jeeri en peul. À ces deux grandes catégories s'ajoutent les Fula6e, grands éleveurs originaires du Bundu, qui dans la première moitié du $\mathrm{Xx}^{\mathrm{e}}$ siècle fréquenteront le sud de l'Aftūt de Mbout. Au lendemain de la Seconde Guerre mondiale, ils se dirigeront à l'est vers le Karakoro pacifié.

32. Les cahiers de recensement sont loin d'être précis mais ils permettent néanmoins de se faire une idée de l'importance du peuplement peul dans la région. 
notamment le cas des Fulbe jeeri Pambinaabe, particulièrement attachés aux Šurvā qui leur concédèrent des terrains aux alentours de Mbout en échange de redevances.

Mais, parfois, les Peuls parvinrent à obtenir des terrains directement du colonisateur. Le cas des Fresbe (Fulbe waalo) est sur ce point remarquable car ils ont été les premiers Peuls à résider à Mbout dès la création du poste en $1904^{33}$. Leur installation fut favorisée par l'obtention de terres concédées par le commandant Dumas. Ce sont le plus souvent les Silah, ces petits territoires annexes des grands, qui furent recherchés par ceux qui étaient en quête de terrains de culture. Ainsi, les Peuls ont-ils pu s'installer à Ndūmrāte et à Ndūla sur le terrain appelé Salḥa Bale. Ailleurs, les Fresbe Bubu Sule ont enregistré deux Silaḥ : Salḥa Kadial et Salhạa Afadyar (1944). Plusieurs autres groupes peuls ont leurs terres inscrites sur le registre foncier de Mbout.

L'acquisition de terres par certains groupes peuls ainsi que le dynamisme dont a fait preuve la jeune tribu des Šurvā dans sa quête de territorialité montrent, une fois de plus, que le colonisateur n'a pas fixé une carte foncière déjà existante, mais a, au contraire, contribué à modifier les rapports politico-territoriaux. Avec la colonisation le pouvoir a changé et avec lui, les comportements fonciers : ceux qui en bénéficient sont sans conteste les marabouts. Mais si la politique foncière du colonisateur suscita des transformations sociopolitiques importantes, par le biais d'affectations et de désaffectations, elle eut, en revanche, peu d'impact sur le système de tenure des terres, ne cherchant pas à bouleverser les rapports sociaux de production.

\section{Territorialité et enjeux sociaux : les revendications foncières des populations serviles}

En enregistrant les terrains au nom des collectivités, conformément au décret du 8 octobre 1925 relatif au régime de constatation des droits coutumiers, le colonisateur s'est mis en difficulté pour appliquer le principe de l'individualisation des terres qui aurait pu avoir des conséquences importantes sur l'émancipation des groupes serviles. Il est vrai que les administrateurs étaient peu enclins à adopter une mesure qui posait la question de savoir à qui, des bidān contrôlant les terres ou des harāținn qui les mettaient en valeur, fallait-il accorder la propriété ? Dans l'ensemble, les autorités coloniales s'abstinrent de répondre et ne cherchèrent pas à désolidariser ces deux groupes. Les mesures qui allèrent dans le sens contraire furent timides et souvent occasionnelles. Elles répondirent surtout aux résistances des populations serviles et à des considérations d'ordre économique. Ce fut le

33. Si le plus souvent les Fulbe waalo ont eu tendance à suivre la progression au nord des Fulbe jeeri en s'installant aux endroits que ces derniers avaient abandonnés (DuboIs 1962), le cas des Fres6e fait figure d'exception puisqu'ils s'implantèrent les premiers à Mbout. 
cas à Mbout où l'administration procéda à partir de 1927 à des redistributions de terres. Mais celles-ci finirent par poser un certain nombre de difficultés qui limitèrent l'action de la France dans sa politique foncière.

\section{L'action de Mbout-Liberté}

Si Mbout est un des rares endroits où le colonisateur est intervenu directement dans la répartition des terres, c'est d'abord en raison des caractéristiques de son peuplement. Peu de temps après sa création, le 9 mai 1904, le poste de Mbout s'est, en effet, transformé en agglomération vers laquelle ont afflué d'énormes contingents de harāṭīn et 'abīd venus se réfugier et/ ou travailler sous l'autorité coloniale ${ }^{34}$. Mbout prend alors de plus en plus l'allure d'un «village de liberté » à l'image de ceux fixés ailleurs dans l'empire colonial (Bouche 1968). Les nouveaux arrivants se mêlèrent aux soldats des troupes de «tirailleurs sénégalais» présents sur place, dont la plupart étaient d'origine bambara ${ }^{35}$. Ce fut, d'ailleurs, un de ces derniers, en la personne de Fabu Kone, qui fut reconnu par les Français comme représentant de la communauté de «Mbout ville» ou «Mbout-Liberté ».

Pour occuper les uns (harāțīn et 'abìd) et peut-être récompenser les autres (anciens combattants), les Français engagèrent une vaste opération de distribution de terres, notamment aux oueds de Mbout, Farkāne et alBatha, domaine contrôlé par les Šurvā. Les terres furent ainsi délimitées et « cédées » conformément à la convention du 5 septembre 1927 :

«Cette convention établissant que les terrains précités sont les propriétés des habitants de Debaye-Liberté, a été adoptée par la majorité des Chorfas et leur "Jemaa". Il a été convenu ce qui suit :

1) Les habitants de M'Bout qui cultivent ces terrains, acceptent d'acquitter chaque année cinq mouds de mil représentant les droits coutumiers dévolus aux Chorfas.

2) Chaque année au mois de novembre, le chef des Chorfas s'adressera à l'autorité administrative pour que soit facilité le recouvrement de ces redevances.

3) Les propriétaires des "Tamourt" (rizières) ne sont redevables d'aucun droit fixe : chacun payera à volonté ce qu'il pourra.

4) Les Chorfas cèdent ces terrains à vie aux habitants de Debaye-Liberté.

Lorsqu'un propriétaire quitte définitivement le pays, son terrain sera acquis au premier possesseur. À la mort d'un propriétaire terrien, son terrain sera légué à ses héritiers (sa progéniture) qui devront continuer à l'exploiter et à payer les droits prescrits $\gg^{36}$.

34. Signalons que certains administrateurs n'hésitaient pas à restituer à leurs maîtres des captifs venus se réfugier près du poste de Mbout.

35. Les familles bambara originaires des régions du Bougouni, de Segou et de Sikasso ont pour nom : Kone, Camara, Mariko, Keita, Traore, Coulibaly, Sangare, Saro.

36. ANM, série $\mathrm{O}^{\circ} 1$. Convention du 5.9.1927. Curieusement, une deuxième version de cette convention existe dans des termes différents. Il ne s'agit plus de «céder à vie », aussi bien aux cultivateurs actuels qu'à leurs héritiers, mais de "prêter à vie » aux seuls cultivateurs de la première génération, le terrain devant être repris par son propriétaire une fois l'agriculteur mort. Une constante revient cependant dans ces deux copies: le paiement de redevances au bénéfice des 
Cette convention cadrait avec la politique de développement des contrats de métayage, préconisés à l'époque par les Français comme moyen d'abolir l'esclavage en douceur tout en fixant une main-d'œuvre sur place et éviter ainsi des départs massifs vers les villes du Sénégal qui auraient été préjudiciables à la mise en valeur de la colonie (Leservoisier 1994a). Le métayage semblait, par ailleurs, contenter les parties concernées. Les Šurvā, détenteurs des droits sur la zone, pouvaient se départir de l'impôt dû sur les terrains prêtés tout en bénéficiant de redevances. Quant aux agriculteurs, l'obtention de droits de culture leur donnait la possibilité de vivre sur place.

Mais les habitants de Mbout-Liberté ne se satisfirent pas longtemps de leur statut de métayer. La plupart d'entre eux (notamment les anciens combattants d'origine bambara) ne se revendiquaient pas harāțīn et encore moins 'abìd. Ils échappaient à la structure sociale régnante et étaient capables de défier le pouvoir traditionnel d'autant qu'ils pouvaient, eux aussi, compter sur le soutien de l'administration coloniale ${ }^{37}$. La convention n'était donc qu'un moyen pour ces habitants de faire une percée régulière dans le territoire puisque la même année, Bakary Torola, un des chefs de «Liberté », déclara des droits sur le terrain de Ndūmrāte, alors qu'il ne fut défriché qu'en 1928 et 1929. En 1943, un litige les opposa aux Šurvā au sujet de Farkāne, terrain exploité par ces derniers avant l'occupation française. Ils furent déboutés par l'administration mais ne reconnurent le jugement que dix ans plus tard $!^{38}$. En revanche, en 1945, ils réussirent à gagner des terrains à ar-Rakna, domaine qui fut partagé entre les Hašim (partie nord) et Fabou Kone (partie sud).

Dans leur quête de territorialité, les gens de «Liberté » surent saisir les opportunités qu'offraient les réglementations foncières du colonisateur. Ainsi, considérant les nouveaux critères d'attribution (immatriculation, exploitation, etc.), ils bénéficièrent des nouvelles terres domaniales que l'autorité coloniale s'était adjugée au nom du principe des «terres vacantes et sans maître »: vacantes parce qu'encore en friche, sans maître parce que n'étant l'objet d'aucune déclaration « légale ». Ce principe, auquel le colonisateur eut rarement recours ailleurs, leur permit de se retrouver propriétaires légaux de terres arables.

Sorfa. Faut-il voir dans ces deux versions une manœuvre délibérée de l'administration pour satisfaire les deux parties?

37. Jusqu'à aujourd'hui, les descendants de ces familles bambara revendiquent leur origine et leur identité d'habitants de Mbout ville, ce qui s'explique en partie par la volonté de ne pas être totalement assimilés à des haräạtīn. Cependant, leur origine bambara ne leur permettait pas d'espérer une grande considération de la part des bidān dont on sait qu'une grande partie de leurs harâțīn est originaire des régions de Nioro, de Kati et de Bougouni, arrachés à leur pays d'origine et vendus à la suite des guerres de Samory, d'El Hajj Umar et de Mamadu Lamine.

38. On peut lire dans le registre foncier de Mbûd au sujet de ce terrain : "Accordé le 17 janvier 1944 après affichage du 3 septembre 1943 au chef Oulad Hachim et non à M'Bout Liberté donc partie à tort. Fait reconnu par Fabou Koné [le chef] lui-même devant le tribunal coutumier le 15 juin $1953 »$ (ibid.). 
L'obstination avec laquelle les habitants de Mbout ont acquis des terres est à la mesure de l'enjeu social que représentait leur inscription sur un territoire. Dans une société où le statut des individus est fortement lié à celui de la terre, celle-ci était en effet la condition de toute indépendance. Elle était aussi un puissant moyen d'identification et de resocialisation pour ces populations serviles (ou assimilées comme telles), qui furent à l'origine arrachées de leur milieu et désocialisées. Leur combat ne manqua pas d'avoir des répercussions sur les harāțīn des villages environnants, comme le constatait, en 1939, le commandant de cercle Castagnet:

«Certaines fractions éprouveraient un malaise avec leurs harratines. Ces derniers, poussés par les anciens tirailleurs et les travailleurs des grands centres, chercheraient à s'affranchir de la tutelle de leurs maîtres, qui se cramponnent désespérement, vivant uniquement des redevances de leurs serviteurs ${ }^{39}$.

La brèche ouverte en 1927 inaugura donc la voie d'un cycle de revendications, de réclamations, mais aussi de conflits. Le fait nouveau, c'est que ces litiges n'opposaient plus seulement différentes tribus, mais mettaient en lice deux légitimités d'appropriation: l'une basée sur le défrichage et la mise en exploitation des terres, l'autre liée au droit collectif de la tribu qui a été reconnue par l'administration lors de l'enregistrement des terrains. Cette double revendication des terres posait le problème des inégalités sociales criantes entre agriculteurs et maîtres, inégalités qui préoccupèrent de plus en plus les administrateurs, il est vrai, moins pour des raisons morales qu'économiques. En 1945, le Résident de Mbout faisait ainsi le constat suivant :

« Tout effort tendant à obtenir des haratines une production accrue, en leur assurant un niveau de vie plus élevé, devra être accompagné de mesures très strictes visant à garantir aux intéressés le fruit de leur travail, et en fait, à les affranchir réellement et non plus par des formules académiques, des servitudes qui pèsent encore sur eux [...]. Il est manifeste et bien compréhensible qu'aucun hartani ne voudra défricher un champ, construire un barrage, planter du coton ou du ricin s'il pense que dès la récolte, la moitié ou les $2 / 3$ du fruit de son labeur seront pris — on peut dire volés par son patron ${ }^{40}$.

Si cette déclaration peut être interprétée comme marquant un désir d'intervention contre le phénomène d'esclavage encore vivace, elle vise aussi et surtout à l'augmentation de la production agricole par voie d'extension des cultures, à une époque qui fut marquée par une grave crise climatique. L'individualisation est alors défendue dans le Plan quinquenal de Mbout du $1^{\text {er }}$ avril 1945 comme le moyen d'assurer le développement économique tout en permettant d'améliorer le statut des plus démunis :

39. Rapport politique 1939, ANM, dossier E2/100.

40. Archives départementales de Mbout, 4 avril 1945. 
«Pour ce qui concerne le régime foncier, l'action doit surtout porter sur l'évolution vers le droit de propriété individuelle. Celle-ci qui réalise indubitablement un progrès considérable est le seul moyen d'obtenir une réelle extension des cultures en mettant le paysan à l'abri des entreprises plus ou moins honnêtes de son maître ${ }^{41}$.

Mais, signe de l'inconstance des politiques, le haut-commissaire de l'AOF semblait contredire, quelques années plus tard, l'objectif du Plan quinquennal de Mbout :

«Il conviendrait donc de confirmer les droits des communautés traditionnelles sur les terres qu'elles utilisent, et en particulier celui d'en répartir l'usage entre leurs membres, mais avec cette réserve très importante que, dans certains cas, un des membres de la communauté qui s'est acquis par son travail continu des droits particuliers pourrait, s'il le désire, sortir de l'indivision et accéder à la propriété individuelle $\gg$.

L'individualisation n'est plus généralisée. Elle ne doit intervenir que dans certains cas. Précisons que la procédure d'individualisation telle qu'elle était envisagée par ce haut-commissaire ne pouvait être suivie d'effet car elle ne prenait en compte ni les moyens de pression dont disposaient les bidān pour préserver l'indivision des terres ni la nature synallagmatique des relations de dépendance entre les harāțīn et leurs maîtres. Réclamer sa part d'un domaine, c'était se condamner à ne plus pouvoir compter sur la relative sécurité et aide matérielle fournie par sa tribu de rattachement. La démarche était d'autant moins envisageable que le décret du 26 juillet 1932 stipulait que seules les personnes ayant « la capacité de passer des actes administratifs » sont admises à requérir l'immatriculation. Et d'ajouter : «Au nom du propriétaire sont admis à requérir l'immatriculation : le tuteur, le curateur et l'administrateur des biens d'un incapable. » Ainsi, la loi excluait la plupart des paysans puisque le milieu ne leur accordait qu'une éducation bien maigre. Elle faisait de la majorité de cette catégorie des assistés, voire des « incapables » nécessitant un intermédiaire.

On le voit, le colonisateur avait une politique ambiguë hésitant entre le respect des coutumes et une politique domaniale interventionniste. La présentation, en 1954, des objectifs de la politique foncière par le hautcommissaire de la France d'outre-mer résume, à elle seule, cette absence de cohésion :

«a) L'appropriation par les collectivités traditionnelles de superficies très vastes risque d'entraver la mise en valeur du territoire en raison de la faible part des terres réservées à la propriété individuelle.

b) Il existe, en outre, des redevances coutumières, antérieures à notre occupation, dont la suppression pourrait entraîner des revendications, génératrices de troubles sociaux. Il paraît en conséquence souhaitable d'éviter un bouleversement trop radical des institutions existantes.

41. Ibid.

42. Archives départementales de Mbout, 25 décembre 1952. 
c) Il est par contre à craindre que le maintien d'un système domanial périmé crée des obstacles à une exploitation rationnelle et plus rentable des ressources du sol. d) Le respect des droits coutumiers doit se concilier avec les nécessités de l'évolution économique et sociale : aménagements ruraux et urbains, mise en culture de zones nouvelles $\gg$.

Les hésitations de l'administration entre la confirmation des droits tribaux et la promotion du droit individuel s'expliquent fondamentalement, en réalité, par la difficulté de déterminer un droit de propriété : celui-ci devaitil être accordé au bénéficiaire du «droit de redevance » ou au bénéficiaire du «droit de culture »? Là, surgit le problème essentiel dont la solution est moins juridique que politique.

Il reste qu'enregistrer les terres au nom d'une tribu donnée, comme l'a fait le colonisateur, et admettre le versement de redevances à une tranche de cette collectivité, équivalait à reconnaître implicitement la suprématie des receveurs sur les donneurs. Cette reconnaissance n'allait pas sans poser à son tour un certain nombre de questions, notamment sur l'attitude du colonisateur vis-à-vis du problème de l'esclavage. La propriété étant généralement collective, les cultivateurs qui sont membres actifs et à part entière de la tribu devaient-ils, selon lui, verser des redevances en tant que non propriétaires ou parce que non libres? Les propos tenus par le capitaine Gilles, en 1936, entretiennent pour le moins une certaine ambiguïté : « Ce qui fait la propriété, c'est le fait de cultiver. Ce qui faisait le droit du Maure sur la récolte, c'est qu'il était le propriétaire de l'esclave qui récoltait ${ }^{44}$. Sans doute, le problème des Français est de n'avoir jamais voulu réellement trancher entre ces deux principes.

Tout au long de son séjour en Mauritanie, le colonisateur a donc été pris dans un engrenage de mécanismes allant du droit de nomadisation à celui du défrichage. Ses décisions furent entachées d'errements, oscillèrent entre deux attitudes : ménager une chefferie alliée et appliquer ses principes « productivistes » et «égalitaires ». L'autorité coloniale, en appliquant sa réglementation foncière, ne voyait que les tribus et non les individus au sein de celles-ci. Elle n'a, de ce fait, jamais cherché à rendre obligatoire l'immatriculation des immeubles, comme le supposait la mise en place d'un cadastre. Rien de surprenant, dès lors, qu'en 1936, un administrateur nous fasse savoir que «bon nombre de champs de cultures ne sont pas immatriculés ${ }^{45}$.

43. ANM, série O 338, 2 juin 1954.

44. ANM, dossier E2/114.

45. ANM, dossier E2 105. Rapport de tournée dans la région de M'Bout, 15 novembre 1936. 
Cependant, en essayant plus ou moins de fixer la carte tribale de la région, le colonisateur y intégra de nouveaux acteurs mais aussi de nouveaux mécanismes de territorialité qui engendrèrent des transformations sociopolitiques importantes dont les tribus guerrières furent les premières victimes. Aussi, de façon sélective, l'agriculture a fait de plus en plus figure de principale forme d'inscription foncière légitime puisque durable. En ce sens, l'héritage principal de la politique foncière du colonisateur dans l'Aftūt de Mbout demeure le registre des terres. On le constate, aujourd'hui, lors des conflits d'appropriation entre harāțīn et bidān. Ces derniers n'hésitent pas à se référer à leurs titres obtenus durant la période coloniale pour faire valoir leur droit et empêcher ainsi que la terre ne sorte du cadre tribal, allant, par ailleurs, jusqu'à mettre en garde contre " les risques d'une éventuelle remise en cause du sacro-saint principe de l'intangibilité des frontières héritées de la colonisation ${ }^{46}$ ! Ainsi, non seulement le registre foncier n'a pas été l'instrument d'une réforme du système de tenure des terres, mais il apparaît aujourd'hui dans certains cas comme un obstacle à l'application de toute nouvelle réforme foncière.

Si l'immatriculation faite au nom des fractions de tribus reste (restera) un référent et une pièce maîtresse dans toute justification de propriété, elle ne constitue cependant pas la seule forme probante de la propriété. Ce droit est en effet sujet à plusieurs interprétations, ce qui le rend flexible en fonction de l'interprétation que l'on veut en faire : jusqu'en 1957, à la veille de l'Indépendance, il s'effectuait de nouveaux enregistrements, de nouvelles possessions ou dépossessions, de nouveaux partages. Il est néanmoins remarquable de voir comment ce registre peut servir certaines tribus à inventer des traditions. Elles revendiquent ainsi une antériorité de l'occupation du sol en prétextant que si leurs terres ont été immatriculées c'est parce qu'elles les occupaient avant la colonisation... C'est oublier que leur inscription territoriale dans la zone fut le plus souvent contemporaine de cette même colonisation.

Université de Nouakchott, Nouakchott.

Université de Paris V, Paris.

46. Entretien avec Abdellahi w Arbi, Nouakchott, le 21 février 1998. 


\section{BIBLIOGR APHIE}

Albergoni, G.

1990 «Les Bédouins et les échanges : la piste introuvable », Cahiers des Siences humaines, 26 (1-2): 195-215.

BA, O.

1969 «Du peuplement peul de la Subdivision de MBout (Mauritanie)», Notes africaines, 124 : 114-118.

BONNET-DUPEYRON, F.

1947 Note sur l'infiltration peule en Mauritanie à l'ouest de l'Assaba, Compte rendu, Conférence internationale Afrique occidentale, vol. V, Bissau, CIAO : $13-26$.

Bouche, D.

1968 Les villages de liberté en Afrique noire française 1887-1910, Paris-La Haye, Mouton.

Cabrol, C.

1954 «Populations peules et sarakollé de la Subdivision de MBout (Mauritanie)», Notes africaines, $21: 2-4$.

DAKHLIA, J.

1990 L'oubli de la cité. La mémoire collective à l'épreuve du lignage dans le Jérid tunisien, Paris, La Découverte.

DARESTE, $\mathrm{P}$.

1908 Le régime de la propriété foncière en AOF, Paris, Marchal \& Billard.

DOUBLIER, R.

1952 La propriété foncière en AOF, Saint-Louis du Sénégal, Imprimerie du gouvernement.

Dubois, J.-P.

1962 La basse vallée du Gorgol, Mauritanie, Saint-Louis, Mission d'aménagement du fleuve Sénégal (Étude de géographie régionale, Bulletin ${ }^{\circ} 133$ ).

ELA, J.-M.

1990 Quand l'État pénètre en brousse... Les ripostes paysannes à la crise, Paris, Karthala.

Flandin, E.

1908 «La politique française en Mauritanie», Bulletin de la Réunion d'Études algériennes, $1: 2-26$.

HERvouËT, J.-P.

1975 Type d'adaptations saheliennes. L'exemple des éleveurs de la Mauritanie centrale méridionale, thèse de $3^{\mathrm{e}}$ cycle, Rouen, Université de Rouen. 
Kamara, C. S. B.

1993 Le foncier rural et le développement en Mauritanie. Perspective historique. L'Aftūt de Mbûd à l'heure du barrage de Foum Laglayta, thèse de Ph. D., Québec, Université Laval.

LESERVOISIER, O.

1994a La question foncière en Mauritanie. Terres et pouvoirs dans la région du Gorgol, Paris, L'Harmattan.

1994b «L'évolution foncière de la rive droite du fleuve Sénégal sous la colonisation (Mauritanie) », Cahiers d'Études africaines, XXXIV (1-3), 133-135: 55-84.

SANTOIR, C.

1990 «Les Peul "refusés", les Peul mauritaniens réfugiés au Sénégal (département de Matam)», Cahiers des Sciences humaines de l'Orstom, 26 (4) : 577-603.

SAUTTER, G.

1982 «Quelques réflexions sur les frontières africaines », Pluriel, 30 : 41-50.

Schmitz, J.

1987 Projet d'irrigation Gorgol noir II. Rapport socio-économique, Paris, ORSTOM, multig.

Villasante Cervello, M.

1989 Collectivités tribales, restructuration des stratégies sociales de reproduction et de pouvoir: quelques aspects du système foncier dans la région de l'Assaba, RIM, mémoire de diplôme de recherche en études du développement, Genève, Institut universitaire d'études du développement.

\section{RÉSUMÉ}

La création par l'administration coloniale française d'un registre foncier en Aftūt de Mbout (Mauritanie) a engendré des transformations sociopolitiques importantes. II en résulte ainsi que loin de fixer une carte foncière déjà existante, le colonisateur a, au contraire, contribué à modifier les rapports politico-territoriaux en intégrant de nouveaux acteurs et en privilégiant de nouvelles formes d'inscriptions territoriales reposant sur la sédentarisation et l'exploitation des terres. De nombreuses tribus maraboutiques comme celle des Šurvā de Mbout sauront tirer profit de ces nouveaux mécanismes de territorialité afin d'acquérir des territoires et asseoir ainsi une domination politique. Cette quête de territorialité sera partagée par la plupart des groupes peuls de la région mais aussi par certains groupes d'origine servile soucieux de faire valoir leurs droits sur les terres. Les revendications de ces derniers placeront les autorités françaises devant la difficulté de déterminer les droits de propriété entre le maître et l'esclave et, plus largement, de décider de la politique à adopter vis-à-vis de I'esclavage.

\section{ABSTRACT}

The Quest for Territoriality in Mbout Aftūt under French Colonization (Mauritania). - The creation by the French colonial administration of a land registry in Mbout 
Aftūt (Mauritania) set off major social and political changes. Far from registering a previously existing state of landholdings, French authorities modified political and territorial relations by integrating new actors and promoting new forms of territoriality based on making people sedentary and working the land. Several marabout tribe, such as Mbout Survā, were able to profit from these new territorial arrangements in order to acquire land and install their political domination. Most Fulani groups in the area took part in this quest for territoriality, as did certain groups of slave origins, who sought to have their land rights recognized. Claims by the latter groups placed French authorities before the difficulty of determining property rights between master and slaves and, more broadly, of making a decision about the policy to adopt toward slavery.

Mots-clés/Keywords : Mauritanie, Šurvā, colonisation, conflits, esclavage, redevances, registre foncier, segmentarité, territorialité, tribu maraboutique/Mauritania, Šurvā, colonial administration, conflicts, land rights, marabout, slavery, territoriality. 